\title{
The rarest of bounties
}

\author{
Brady Huggett
}

In a land where drugs for ultra-rare indications are the new blockbusters, Alexion's Soliris is king.

ast year, Alexion Pharmaceuticals reported Lmodest net income of about \$255 million. This would hardly seem to mark Alexion as a biotech star, yet the company's stock ended 2012 at $\$ 93.74$, with a market cap greater than $\$ 18$ billion. Alexion thus ranked in market cap right below biotech bellwether Biogen Idec, which for 2012 reported net income of about $\$ 1.4$ billion, fifth highest of today's public biotechs. In July rumors surfaced that Alexion might be bought, with analysts speculating the price-per-share for any purchase could climb as high as $\$ 130$.

What has given Alexion these lofty valuations?

The company was founded in 1992 to pursue complement inhibition therapies. Early on, it developed the humanized monoclonal antibody Soliris (eculizumab), which is designed to block the production of C5a and C5b-9, both mediators of the inflammatory process. After false starts testing the drug in asthma and autoimmune disorders, the company launched a trial in 2002 to test the use of Soliris in the fatal condition paroxysmal nocturnal hemoglobinuria (PNH). It would go on to run a pilot phase 2 study in $\mathrm{PNH}$, and two phase 3 trials (TRIUMPH and SHEPHERD). Of the 195 people enrolled in those trials, everyone who received Soliris had an objective improvement in hemolysis, and reductions in blood transfusions, anemia and the risk for thrombosis. Gauging these impressive data, Alexion knew it had a gold mine on its hands.

And it has mined it. In 2007, Soliris became the only drug approved to treat PHN to reduce hemolysis, and Alexion aggressively priced it at around $\$ 400,000$ annually, giving it the dubious honor of being the world's most expensive drug. (Alexion provides the drug free to those who cannot afford it.) A second indication came in 2011: atypical hemolytic uremic syndrome (aHUS). Here, too, Soliris is the first and only drug approved to treat a small but needy patient population. Dosing is slightly higher in

Brady Huggett is Business Editor at Nature Biotechnology. this indication, which pushes the annual price tag to around $\$ 450,000$. The company reported net sales of $\$ 1.13$ billion in 2012.

Charging a premium for efficacious drugs in unmet niche indications is not newGenzyme paved this business path long ago. Its first orphan drug, Ceredase (alglucerase), was approved in 1991 for Gaucher disease, and the second-generation drug, Cerezyme (imiglucerase), in 1994. The company charged around $\$ 350,000$ per year per patient, and through the years it brought in billions of dollars.

Genzyme's success produced business model copycats, such as BioMarin, which was founded in 1997. Like Genzyme, BioMarin focuses on enzyme-replacement therapies for niche indications and charges premium prices. Its four approved products-Naglazyme (galsulfase), Kuvan (sapropterin dihydrochloride), Aldurazyme (laronidase) and Firdapse (approved only in EU; amifampridine phosphate) - earned $\sim 497$ million in net revenue in 2012.

But Alexion, of Cheshire, Connecticut, has surpassed these successes in two ways. First, it has been exceptionally effective at simultaneously controlling costs and casting a wide net for rare patients. Last year, it reported cost of sales of $\sim \$ 126$ million (representing only $11 \%$ of net product revenue-a low figure for producing a biologic). The company has kept costs around this level since the drug was introduced, which means that even as Alexion expands its global reach-it has operations in 50 countries and is earmarking such areas as Korea, Turkey and Latin America for expansionthe bottom line has held steady.

In comparison, when Genzyme introduced Cerezyme, it had net product sales of $\sim \$ 72$ million against a cost of those products sold of $\$ 33.2$ million, or $46 \%$. This has improved over the years (but was still $29 \%$ in 2010, the last full year of reporting before Sanofi bought it). BioMarin has done better-its cost of products sold was $18 \%$ in 2012-but that still cannot touch Alexion. Whether the reason is company size; an easier, high-yielding manufacturing process; a focused sales force; or Soliris's higher

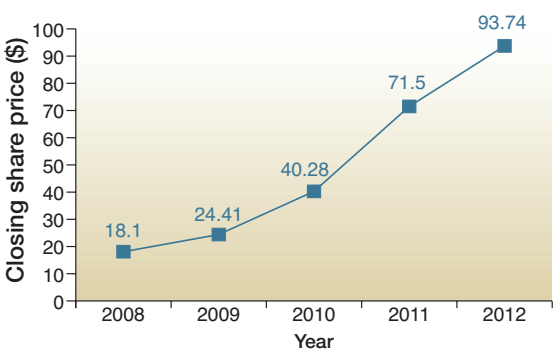

Figure 1 The rise of Alexion stock.

price, Alexion is pocketing more per sale than others.

Second, Alexion's research has been remarkably serendipitous. In Soliris, it has the rarest of rare things - an ultra-orphan drug that works exceptionally well in another orphan indication lacking treatment options. Net sales for Soliris last year were up 45\% from 2011, and though sales were heavily slanted to the more established PNH indication, the company believes the aHUS indication will eventually be as large as $\mathrm{PNH}$ and is currently investigating nine other indications-no wonder investors are salivating. In contrast, the orphan indication enzyme replacement therapies of Genzyme and BioMarin are lifesaving and valuable revenue drivers, but they are one-trick ponies.

Of course, there are threats to Alexion's premium pricing model. It is currently awaiting review by the UK National Institute for Health and Care Excellence and other reimbursement authorities across the broader European Union. And skeptics warn that this rapid patient discovery and revenue growth cannot continue.

For now, though, Alexion's stock is on a steep upward climb (Fig. 1). And the company has diversified through the acquisition of Enobia in 2011, bringing aboard ENB-0400 (asfotase alfa), an enzyme replacement therapy for the ultrarare, genetic metabolic disease hypophosphatasia. The move oozes synergy. But it would be foolish to expect that drug - or any other for that matter-to perform like Soliris. Soliris is a oncein-a-lifetime drug for the patients it helps, and it's a once-in-a-lifetime drug for the company it buoys. 